\title{
Characterizing the relationship between functional MRI-derived measures and clinical outcomes in patients with vascular lesions
}

\author{
*Thomas A. Gallagher, M.D., ${ }^{1}$ Veena A. Nair, Ph.D., ${ }^{2}$ Michael F. Regner, B.A., 2 \\ Brittany M. Young, B.S., ${ }^{2}$ Andrew Radtke, B.A., ${ }^{2}$ Joshua Pankratz, B.S., ${ }^{2}$ \\ Ryan L. Holdsworth, M.D., ${ }^{2}$ Dovile Baniulis, B.S., ${ }^{2}$ NiCOle K. Kornder, B.S., ${ }^{2}$ \\ Jed Voss, B.S., ${ }^{2}$ Benjamin P. Austin, Ph.D., ${ }^{3}$ Chad Moritz, B.S., R.T., 2 \\ Mary E. Meyerand, Ph.D., ${ }^{4}$ and Vivek Prabhakaran, M.D., Ph.D. 2 \\ ${ }^{1}$ Department of Radiology, Feinberg School of Medicine, Northwestern University, Chicago, Illinois; and \\ ${ }^{2}$ Department of Radiology, School of Medicine and Public Health, ${ }^{3}$ Cardiovascular Research Center, and \\ ${ }^{4}$ Department of Medical Physics, University of Wisconsin-Madison, Wisconsin
}

Object. Functional MRI (fMRI) has proven to be an effective component of pretreatment planning in patients harboring a variety of different brain lesions. The authors have recently reported significant relationships concerning distances between brain tumor borders and areas of functional activation (lesion-to-activation distance; LAD) with regard to patient morbidity and mortality. This study further examines the relationship between LAD, focusing on a host of vascular lesions and pre- and posttreatment morbidity.

Methods. This study included a sample population of patients with vascular lesions $(n=106)$, primarily arteriovenous malformations (AVMs) and cavernomas. These patients underwent pretreatment fMRI-based motor mapping $(n=72)$ or language mapping $(n=84)$. The impact of LAD and other variables derived from the patient medical record were analyzed with respect to functional deficits in terms of morbidity (weakness and/or aphasia).

Results. In patients with no pretreatment deficits, there was trend for a significant relationship between the Wernicke area LAD and posttreatment language deficits. In patients with or without pretreatment deficits, a trend toward significance was observed between sensorimotor LAD and posttreatment motor deficits. Additionally, lesion type (AVMs or cavernomas) affected posttreatment deficits, with more patients with cavernomas showing posttreatment language deficits than patients with AVMs. However, this difference was not observed for posttreatment motor deficits.

Conclusions. These findings suggest that the proximity of a vascular lesion to sensorimotor and language areas is a relevant parameter in estimating patient prognosis in the perioperative period. Additionally, vascular lesion type and existence of pretreatment deficits play a significant role in outcomes.

(http://thejns.org/doi/abs/10.3171/2013.2.FOCUS12417)

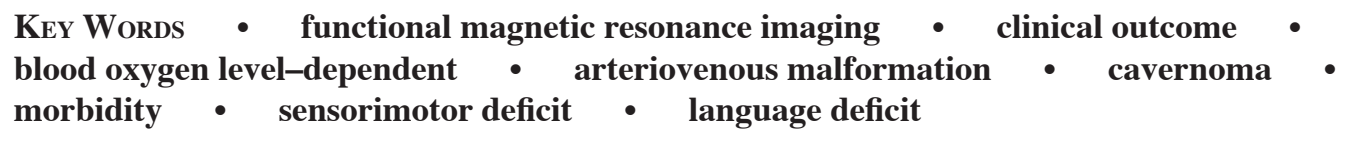

$\mathrm{B}$ LOOD oxygen level-dependent fMRI ${ }^{11,12}$ has gained acceptance as an effective, noninvasive method of brain mapping for pretreatment planning..$^{13}$ In particular, it has been demonstrated to be an effective means to establish language hemisphere dominance, ${ }^{2,15,22}$ and its rendering of functional anatomy has correlated well with direct cortical stimulation mapping., ${ }^{3,8,14,21}$ For patients

\footnotetext{
Abbreviations used in this paper: AVM = arteriovenous malformation; $\mathrm{BOLD}=$ blood oxygen level-dependent; $\mathrm{df}=$ degrees of freedom; fMRI = functional MRI; LAD = lesion-to-activation distance.

* Drs. Gallagher and Nair contributed equally to this work.
}

about to undergo treatment for various vascular brain lesions, however, fMRI presents unique challenges to the investigator. ${ }^{7,18}$ Radiological examination of eloquent cortex utilizing principles of BOLD contrast in the vicinity of a lesion such as an AVM that features abnormal blood flow requires scrutiny of the reliability of activation and overall interpretation of results. Furthermore, given the sensitivity of echo planar imaging to areas of parenchymal hemosiderin staining, vascular brain lesions such as cavernomas with previous hemorrhage may feature accelerated T2 shortening and T2* susceptibility effects that could compromise or render the regional BOLD signal unattainable. Thickbroom et al. ${ }^{18}$ determined that 
flow-related effects were less important than a susceptibility artifact from hemosiderin staining, because the latter could contribute to possible underestimation of the extent of true activation. However, Lehéricy et al. ${ }^{7}$ suggested that AVMs resulting in severe flow anomalies could potentially compromise the reliability of the BOLD signal in a region of interest.

The importance of delineating areas of eloquent cortex remains an important goal for both clinicians and neuroradiologists. The compromise of eloquent areas, as determined either by localizing the clinical deficits or based on conventional imaging, has significant implications for treatment and prognosis. Specific grading schemes for AVMs such as the widely used Spetzler-Martin criteria ${ }^{17}$ classically take into account AVM nidus measurement, location, and organization of venous drainage (Table 1). Recently, additional prognostic characteristics of AVMs have been added to this list that have been termed angioarchitectual weak points. ${ }^{6}$ These weak points may confer greater propensity for hemorrhage and posttreatment risks including presence of a nidal aneurysm, venous stenosis or ectasia, deep venous drainage, isolated or single venous drainage, and posterior fossa location. Although some of these features are not specifically included in the classic Spetzler-Martin grading system, it is important to consider these variables because of their prognostic value and impact on treatment strategies. For example, nidal aneurysms, particularly those exceeding $5 \mathrm{~mm}$, may be treated using coil embolization prior to resection of the AVM. Furthermore, identification and preservation of en passant arteries, which might supply normal brain as well as an AVM, are important tasks.

Currently, imaging examinations in conjunction with a clinical examination provide the diagnostic basis for a vascular lesion such as an AVM or cavernoma. There is a paucity of literature describing the utility of fMRI in routine pretreatment assessment of patients with vascular lesions. Functional MRI has been shown to be a robust technique for establishing language hemisphere domi-

\section{TABLE 1: Classic Spetzler-Martin grading of AVMs*}

\begin{tabular}{lc}
\hline Characteristic & Score \\
\hline size of nidus $(\mathrm{cm})$ & 1 \\
$<3$ & 2 \\
$3-6$ & 3 \\
$>6$ & \\
eloquent cortex $\dagger$ & 0 \\
no & 1 \\
yes & \\
venous drainage & 0 \\
superficial (all cortical) & 1 \\
deep &
\end{tabular}

* Summation of characteristics of a particular AVM determines grade and estimates surgical risk.

$\dagger$ Eloquent cortex constitutes sensorimotor, language, and visual cortex, as well as the internal capsule, thalamus, hypothalamus, cerebellar peduncles, brainstem, and deep cerebellar nuclei. nance, as well as a useful technique for identifying eloquent cortex to be spared during surgery. However, the role of fMRI in the special context of a vascular lesion has yet to be determined. This situation is further complicated by the fact that patients with vascular lesions, including those examined prior to clinical intervention, may have atypical language networks. ${ }^{19}$

In the present study we investigated fMRI LAD and its relationship to morbidity in patients with vascular brain lesions, primarily AVMs and cavernomas, about to undergo treatment. We hypothesized that as the LAD decreases, overall morbidity will increase.

\section{Methods}

\section{Patient Characteristics}

This study was reviewed and approved by the Health Sciences Institutional Review Board of the University of Wisconsin-Madison. The sample population of patients $(\mathrm{n}=106)$ underwent pretreatment fMRI between June 1999 and July 2011 for various vascular brain lesions, including AVMs and cavernomas. Demographic information of the patient population is presented in Table 2. Patients gave informed consent to participate in the study in accordance with the study protocol approved by the institutional review board. Patients' clinical information was extracted from the electronic medical record. Any record of pretreatment or posttreatment motor weakness (upper extremity, lower extremity, or facial weakness) or aphasia (Broca type, Wernicke type, conduction, or global aphasia) was included in the analysis. Only gross motor and language deficits were considered and no specific neuropsychological testing was included. Vascular

TABLE 2: Characteristics of patients with vascular lesions near the primary sensorimotor, Broca, and Wernicke areas according to LAD

\begin{tabular}{|c|c|c|c|c|}
\hline \multirow[b]{2}{*}{ Area of Activation } & \multicolumn{3}{|c|}{$\mathrm{LAD}(\mathrm{mm})$} & \multirow[b]{2}{*}{ p Value } \\
\hline & $<10$ & $10-20$ & $>20$ & \\
\hline \multicolumn{5}{|l|}{ primary sensorimotor } \\
\hline$\%$ male & 54 & 62 & 40 & 0.19 \\
\hline mean age (yrs) & 31 & 34 & 38 & 0.38 \\
\hline$\%$ rt handedness & 54 & 76 & 64 & 0.68 \\
\hline$\%$ w/ AVM & 54 & 50 & 47 & 0.90 \\
\hline \multicolumn{5}{|l|}{ Broca } \\
\hline$\%$ male & 0 & 46 & 49 & 0.60 \\
\hline mean age (yrs) & 20 & 37 & 37 & 0.47 \\
\hline$\%$ rt handedness & 0 & 55 & 65 & 0.47 \\
\hline$\%$ w/ AVM & 100 & 27 & 49 & 0.23 \\
\hline \multicolumn{5}{|l|}{ Wernicke } \\
\hline$\%$ male & 0 & 25 & 51 & $0.05^{*}$ \\
\hline mean age (yrs) & 30 & 31 & 38 & 0.14 \\
\hline$\%$ rt handedness & 25 & 58 & 62 & 0.75 \\
\hline$\%$ w/ AVM & 25 & 58 & 47 & 0.49 \\
\hline
\end{tabular}

* Statistically significant. 


\section{Functional MRI-derived measures and outcomes in vascular lesions}

lesions were diagnosed using catheter cerebral angiography, conventional CT angiography, or MRI/MR angiography. Lesions were categorized as encroaching on sensorimotor cortex or primary language centers (the Broca or Wernicke areas). Functional paradigms were selected depending on the location of the vascular lesion in question, with the aim of eliciting either primary sensorimotor or language center activation. ${ }^{9}$ Functional MRI activations were individually thresholded at the time of the radiological examination in question and then made available for clinical use and decision-making; these same thresholded maps were used retrospectively in this study for distance measurements to characterize the prognostic utility of measures derived from these clinical fMRI maps. Given the wide variation in appearance and distribution of many vascular lesions, distance to the closest presumed edge of a vascular nidus was used. If a compact vascular nidus could not be definitively identified, distance to closest involved component of the vascular lesion was used. Distances from vascular lesion edge to the periphery of fMRI activation and distances from the the vascular lesion edge to the center of maximum primary motor or language fMRI activation were both measured. These distances were then correlated with pretreatment and posttreatment morbidity information obtained from the electronic medical record.

\section{Functional MRI Language Paradigms}

The language paradigms used to assess patients are described in more detail in Moritz and Haughton. ${ }^{9}$ In brief, activation of the Broca area was best observed using word generation fMRI tasks. Two word generation tasks were used: 1) alternating 20-second blocks of antonym word generation and rest, and 2) alternating 20 -second blocks of letter word generation task and rest. In the antonym word generation the patient is asked to silently think of the opposite of the word displayed on the screen. In the letter word generation task, the patient is asked to silently think of words starting with the letter displayed on the screen. The Wernicke area was identified with alternating 20-second blocks of a text reading and symbols task. In this task, the patient silently read a short paragraph in the text reading block. During the control block, the patient was shown a paragraph of symbols and asked to scan for specific symbols within the paragraph. The control block controlled for eye movements during reading, which helped discriminate visual and eye movement-related activity from the true language areas. Not all patients performed all tasks. Tasks were individualized to the patient so that clinically useful and robust language-related activation could be imaged.

\section{Functional MRI Acquisition and Processing}

Radiological examination was performed with either a 1.5- or 3-T commercial MRI machine (GE Medical Systems) equipped with high-speed gradients. The BOLDweighted single-shot echo planar images were obtained continuously for each patient during task performance. The technical parameters used in imaging were as follows: TR $2000 \mathrm{msec}$, TE $40 \mathrm{msec}$ (for $1.5 \mathrm{~T}$ ), TE $27 \mathrm{msec}$ (for $3 \mathrm{~T}$ ), FOV $24 \mathrm{~cm}$, matrix $64 \times 64$, FA 85 (for $1.5 \mathrm{~T}$ ), FA 75 (for $3 \mathrm{~T}$ ), 6-mm coronal plane sections (for $1.5 \mathrm{~T}$ ), and 5-mm axial plane sections (for $3 \mathrm{~T}$ ). Spatial coverage was sufficient to provide mapping of the entire cortex. The number of images and length of imaging varied with the paradigm used. The duration of MRI examination ranged from 3 to 5 minutes. Additional high-resolution anatomical scans, including 3D volumetric T1- and T2weighted contrast sequences, were acquired as part of the pretreatment assessment. Postprocessing of the echo planar imaging signal included both spatial and temporal smoothing. The 3Dvolreg program within the AFNI software was used to correct for head motion in the reconstructed time courses. The echo planar images were spatially coregistered with structural images. Activation was determined by cross-correlation of the time course of the echo planar imaging signal at each voxel with a generalized least-squares fitting algorithm to a smoothed and temporally delayed boxcar reference function, modeling the presumed hemodynamic response. This comparison provided a voxel-wise t-statistic, which was used to individually threshold images to optimize visualization of motor and language areas, and was overlaid on the spatially coregistered anatomical brain volume maps. Thresholding was applied to each individual fMRI examination by an expert clinical fMRI technician and was verified by a diagnostic neuroradiologist with the intent of optimizing specificity and sensitivity of task response while minimizing artifacts such as spurious correlations due to head motion. For example, in a data set that exhibited significant task-correlated head motion, it may not have been possible to minimize the artifacts while retaining a sufficient sensitivity to the task-related responses. The task-related response magnitudes were also dependent on factors such as the patient's ability to perform a particular fMRI task or whether the BOLD response was compromised by presence of the vascular lesion in question. Thus, thresholds were individually varied for each fMR image by the attending neuroradiologist based on the quality of the data and mapping concerns relevant to neurosurgical planning.

\section{Data Measurements}

Both right and left hemisphere lesions were included in the analysis. The distance between the lesion border and the area of functional activation (the centroid, or "hot spot") was measured (LAD). Lesions contralateral to the language or motor center in the dominant hemisphere were considered to have an LAD $>2 \mathrm{~cm}$ by default. The edge of an AVM was defined as the nidus, or the closest most focal network of enhancing flow voids. If a clear nidus was not distinct, the edge of the AVM was defined as the closest abnormal enhancing flow void of the lesion. For cavernomas, the lesion edge was defined as the thin margin of the characteristic hemosiderin ring. For computing lesion volume, measurements on the structural scans were recorded in the transverse (x), anterior/posterior (y), and superior/inferior (z) axes in the Picture Archiving and Communication System. Cavernoma volume was calculated using the equation $\left(x^{*} y^{*} z\right) / 2$ where $x, y$, and $\mathrm{z}$ were the maximum dimensions of the lesion along 
the $\mathrm{x}, \mathrm{y}$, and $\mathrm{z}$ axes, respectively. ${ }^{10}$ The $\mathrm{T} 2 *$ sequences were not used in measurements to avoid error introduced by confounding blooming artifacts. As a control comparison, 20-second fMRI acquisitions with breath holding were obtained to delineate areas of the brain capable of producing measurable BOLD signal. Examples of typical measurements are depicted in Fig. 1.

\section{Statistical Analyses}

All statistical analyses were performed using the statistical package SPSS (version 20.0.0, IBM Corp.). Categorical variables (such as sex and handedness) were compared between groups using chi-square tests. Age, a continuous measure, was compared using the KruskalWallis 1-way ANOVA test (nonparametric equivalent of 1-way ANOVA). The LAD was analyzed as a categorical variable (distance between lesion edge and activation centroid $<10 \mathrm{~mm}$, between 10 and $20 \mathrm{~mm}$, and $>20 \mathrm{~mm}$ ). Multiple logistic regression models with LAD as the predictor and posttreatment motor and language deficits as outcome measures were tested to investigate if LAD was a significant predictor of posttreatment deficits. Because there was a significant sex difference among the patients when grouped by Wernicke LAD, sex was included as a predictor in the regression model for Wernicke LAD. Results were considered statistically significant if $p<0.05$ and as showing a trend toward significance if the $p$ value

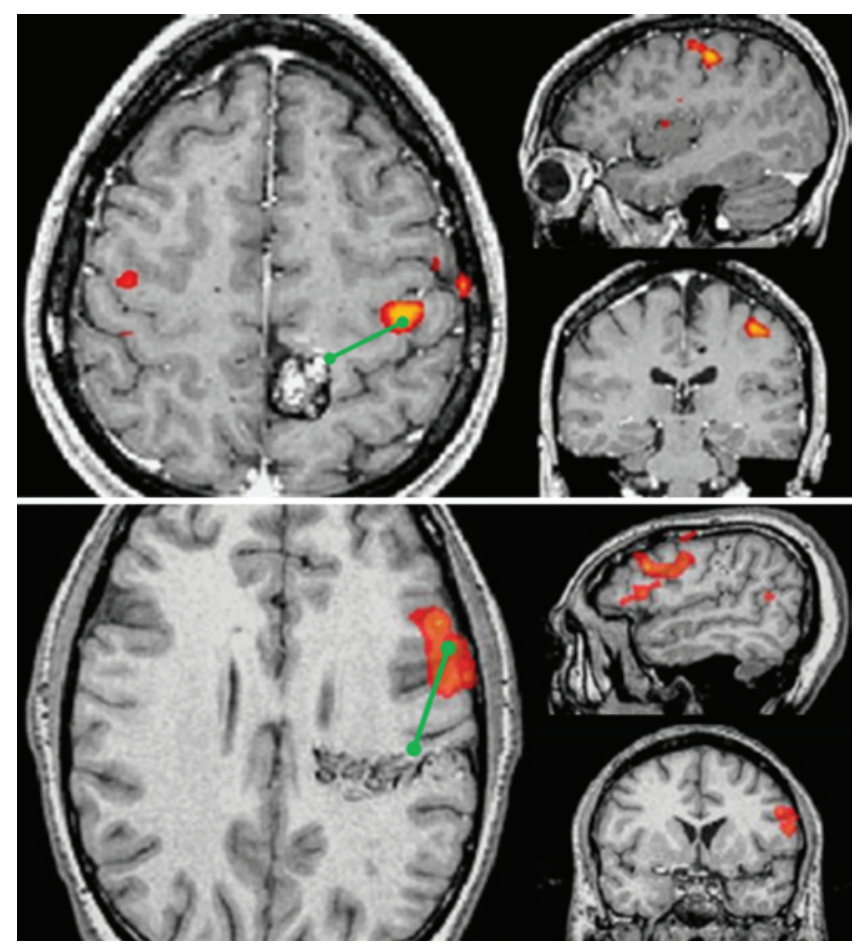

FIG. 1. Axial, sagittal, and coronal MR images showing typical LAD measurements. Upper: Images in a patient showing left sensorimotor activation (orange and red areas) in close proximity to a posterior left frontal cavernoma. Lower: Images in a patient with a left frontal AVM showing Broca area activation during antonym word generation task (orange and red areas). The LADs (green lines) are shown from lesion edge to activation centroid as measured in the Picture Archiving and Communication System. was between 0.05 and 0.10 . Results across all patients are first described below, followed by results from separate analyses for patients with AVMs and cavernomas.

\section{Results}

\section{Demographic Variables}

Patients harboring vascular lesions were studied with regard to proximity of the lesion to primary motor cortex $(n=72)$ and primary language areas $(n=84)$. When grouped by LAD, there were no significant differences based on age or handedness (Table 2). There was a statistically significant difference $(p=0.05)$ between sex and Wernicke LAD. When grouped by presence or absence of posttreatment sensorimotor deficits, there were no significant differences based on sex, age, or handedness (Table 3). Table 4 similarly shows patient characteristics when grouped by posttreatment language deficits.

\section{Types of Lesions}

In this study there were 68 AVMs (64\%), 33 cavernomas $\left(31 \%\right.$, with an average size of $\left.2.8 \mathrm{~cm}^{3}\right)$, and 5 other types of lesions (5\%, those with an unclear diagnosis such as cavernoma/AVM or intracerebral hemorrhage/hemorrhagic lesion). The Spetzler-Martin grade of patients grouped by proximity of the lesion to the Wernicke or Broca areas or the sensorimotor cortex is shown in Table 5. Lesion size and total lesion burden varied from patient to patient, as did history of sentinel hemorrhage, seizure, and pretreatment language or motor deficit. While most patients with cavernomas had a single lesion, 1 patient demonstrated multiple cavernomas, and the lesion closest to the area of eloquent cortex in question was used in the analysis. For the 106 patients in the study, 51 (48\%) underwent resection, $16(15 \%)$ underwent radiation therapy, $23(22 \%)$ had embolization, and $16(15 \%)$ received conservative treatment. Patients with cavernomas underwent either resection or conservative management. Patients with AVMs may have received any of the different treatment types as well a combination of invasive and noninvasive treatments.

\section{Transient or Persistent Deficits}

A record of pretreatment and posttreatment deficits

TABLE 3: Characteristics of patients with vascular lesions near the primary sensorimotor area, grouped by presence or absence of posttreatment sensorimotor deficits (weakness)

\begin{tabular}{lccc}
\hline \multicolumn{1}{c}{ Characteristic } & No Weakness & Weakness & p Value \\
\hline$\%$ male & 46 & 48 & 0.56 \\
mean age (yrs) & 37 & 37 & 0.64 \\
$\%$ rt handedness & 80 & 80 & 0.83 \\
lesion type* & & & 0.17 \\
$\%$ w/ AVM & 60 & 78 & 0.21 \\
$\%$ w/ cavernoma & 35 & 17 & 0.13 \\
\hline
\end{tabular}

* $p$ value for main effect of lesion type on presence or absence of posttreatment sensorimotor deficits. 
Functional MRI-derived measures and outcomes in vascular lesions

TABLE 4: Characteristics of patients with vascular lesions near Broca or Wernicke areas, grouped according to presence or absence of posttreatment language deficits (aphasia)

\begin{tabular}{lccc}
\hline \multicolumn{1}{c}{ Characteristic } & No Aphasia & Aphasia & p Value \\
\hline$\%$ male & 49 & 38 & 0.23 \\
mean age $(\mathrm{yrs})$ & 37 & 38 & 0.31 \\
\% rt handedness & 79 & 84 & 0.84 \\
lesion type* & & & 0.59 \\
$\%$ w/ AVM & 65 & 62.5 & 0.34 \\
$\%$ w/ cavernoma & 29 & 37.5 & 0.81 \\
\hline
\end{tabular}

* $p$ value for main effect of lesion type on presence or absence of deficits.

was made from clinician's notes of follow-up visits, wherever available. Transient deficits were defined as deficits that resolved within 6 months. Of the 24 patients with posttreatment aphasia (with or without prior deficits), 19 had transient deficits $(<6$ months since the date of first treatment) and 5 had persistent deficits (lasting $>6$ months since the date of first treatment). Of the 23 patients with posttreatment weakness and/or motor impairments (with or without prior deficits), 19 had transient deficits and 4 had persistent deficits. Details regarding pretreatment and posttreatment deficits in patients with or without prior deficits, with vascular lesions in proximity to sensorimotor or language areas, are shown in Table 6. Only 2 patients presented with no prior language deficits but experienced a posttreatment deficit; 1 patient's deficit resolved within 6 months, whereas the second patient's deficits persisted even at the 12-month follow-up visit. Both of these patients with persistent deficits had cavernomas. One patient with an AVM presented with no pretreatment motor deficit, but showed a posttreatment deficit, and his deficits persisted at 6 months' follow-up.

\section{Patients With No Pretreatment Deficits}

There were no significant differences $(p=0.78)$ in patients without any pretreatment motor deficits when grouped by sensorimotor cortex LAD. Only 1 patient presented with no motor deficit at pretreatment and experienced a posttreatment deficit, and his deficits persisted at the 6-month follow-up evaluation. When grouped by Broca area LAD, there were no significant differences $\left(\chi^{2}\right.$ $[\mathrm{df}=2, \mathrm{n}=72]=2.64 ; \mathrm{p}=0.10)$. When grouped by Wernicke area LAD, there was a trend toward significance $\left(\chi^{2}\right.$

TABLE 5: Spetzler-Martin grade for patients with AVMs near the Wernicke or Broca areas or the sensorimotor cortex

\begin{tabular}{cccc}
\hline $\begin{array}{c}\text { Spetzler-Martin } \\
\text { Grade }\end{array}$ & $\begin{array}{c}\text { Near Wernicke } \\
\text { Area (\%) }\end{array}$ & $\begin{array}{c}\text { Near Broca } \\
\text { Area (\%) }\end{array}$ & $\begin{array}{c}\text { Near Sensorimotor } \\
\text { Cortex (\%) }\end{array}$ \\
\hline 1 & 5 & 13 & 7 \\
2 & 43 & 39 & 34 \\
3 & 19 & 13 & 23 \\
4 & 33 & 35 & 33 \\
5 & 0 & 0 & 3 \\
\hline
\end{tabular}

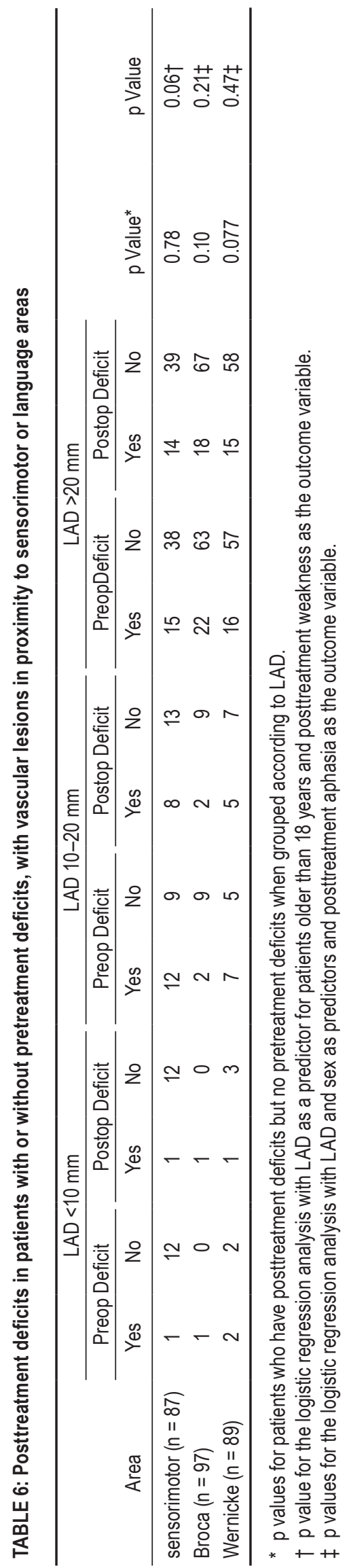


$[\mathrm{df}=2, \mathrm{n}=64]=5.12 ; \mathrm{p}=0.077$ ), with patients in the < $10-\mathrm{mm}$ and the $10-20 \mathrm{~mm}$ categories more likely to show greater posttreatment language deficits in comparison with LAD > $20 \mathrm{~mm}$.

\section{Patients With AVMs}

In patients with AVMs grouped by LAD, there were no significant differences based on sex for sensorimotor cortex $(\mathrm{p}=0.40)$ or the Broca area $(\mathrm{p}=0.43)$, and a trend toward significance for the Wernicke area $(p=0.09)$. There were no significant differences based on handedness (SMC, $\mathrm{p}=0.13$; Broca area, $\mathrm{p}=0.48$; Wernicke area, $p=0.90)$. Additionally, there were no significant differences based on age for sensorimotor cortex $(p=0.49)$ and the Broca area $(\mathrm{p}=0.15)$, but a trend toward significance for the Wernicke area $(\mathrm{p}=0.09)$. Of the 50 patients with AVMs free of pretreatment language deficits, none had any posttreatment language deficits. Of the 49 patients with AVMs free of pretreatment motor deficits, 1 patient experienced a posttreatment motor deficit (Table 7).

\section{Patients With Cavernomas}

In patients with cavernomas grouped by LAD, there were no significant differences based on sex (sensorimotor cortex, $\mathrm{p}=0.25$; Broca area, $\mathrm{p}=0.26$; Wernicke area, $\mathrm{p}$ $=0.32$ ), handedness (sensorimotor cortex, $\mathrm{p}=0.81$; Broca area, $\mathrm{p}=0.15$; Wernicke area, $\mathrm{p}=0.82$ ), and age (sensorimotor cortex, $\mathrm{p}=0.17$; Broca area, $\mathrm{p}=0.48$; Wernicke area, $p=0.59)$. Of the 23 patients with cavernomas free of pretreatment language deficits, 2 had posttreatment deficits. Of the 25 patients free of pretreatment motor deficits, none had any posttreatment deficit (Table 7).

\section{Arteriovenous Malformations Compared With Cavernomas}

For patients with no pretreatment deficits, the chisquare test showed that the difference in posttreatment language deficit by type of lesion, whether an AVM or cavernoma, was statistically significant; more patients with cavernomas had posttreatment language deficits than patients with AVMs $\left(\chi^{2}[\mathrm{df}=1, \mathrm{n}=73]=4.47\right.$; $\mathrm{p}$ $=0.03$ ). The difference in posttreatment motor deficit by type of lesion, whether an AVM or cavernoma, was not statistically significant ( $\mathrm{p}=0.47$; Table 7).

\section{Multivariate Analysis}

Multivariate logistic regression analysis showed that the distance from lesion edge to sensorimotor cortex was not a significant predictor of posttreatment deficits $(\mathrm{p}=$
0.11). In a subset of patients at least 18 years old, there was a trend toward a significant association between distance from vascular lesion to motor activation and the existence of weakness or paresis $(p=0.06)$. A similar analysis was conducted using the Broca and Wernicke areas, which showed that distance from lesion edge to these language areas was not a significant predictor of posttreatment deficits $(p=0.21$ and $p=0.47$, respectively; Table 6$)$. A model combining distance and sex also demonstrated that these were not significant predictors of posttreatment aphasia.

Because age and sex showed a trend toward significance for patients with AVMs, a multivariate logistic regression model was tested with LAD as a predictor and age and sex as covariates. The LAD was not a significant predictor of posttreatment motor or language deficits ( $p$ values for sensorimotor cortex and the Broca and Wernicke areas were $0.36,0.26$, and 0.35 , respectively). A similar analysis was not performed for cavernomas given the smaller patient sample.

\section{Discussion}

Functional MRI is increasingly used for neurosurgical planning due to its ability to provide valuable information on the spatial relationships between intracranial lesions and functionally eloquent areas. The validity of these imaging studies has been demonstrated to be sensitive and specific for mapping language and motor functions; for example, Bizzi et al. ${ }^{3}$ found that fMRI exhibited high sensitivity (83\%) and specificity (83\%) for mapping functional cortical areas, with motor function mapping more sensitive and specific than language function mapping. While there are many studies exploring the value of its use in brain tumor patients, there is a paucity of literature exploring the use of fMRI in patients with AVMs and cavernomas. ${ }^{5}$ To date, there are no studies reporting an association between vascular lesion LAD and clinical outcomes. This information may be useful both to inform patients about their prognosis and to aid in surgical planning.

The present study investigated the clinical outcomes associated with BOLD fMRI parameters measured in patients with vascular lesions. We searched for possible confounds between vascular lesion proximity and demographic characteristics and found no statistically significant relationships. The relationships between LAD and weakness or paresis were investigated. Whereas we found a weak significant relationship between clinical deficits and lesion proximity to functionally eloquent cortex, it did not exist for all cortical areas. Considering both Broca

TABLE 7: Posttreatment deficits in patients with no pretreatment deficits according to lesion type

\begin{tabular}{|c|c|c|c|c|c|c|}
\hline \multirow[b]{2}{*}{ Lesion Type* } & \multicolumn{3}{|c|}{ Motor Deficit } & \multicolumn{3}{|c|}{ Language Deficit } \\
\hline & No & Yes & p Value & No & Yes & p Value \\
\hline AVM & 48 & 1 & 0.47 & 50 & 0 & 0.03 \\
\hline cavernoma & 25 & 0 & & 21 & 2 & \\
\hline other† & 1 & 0 & & 2 & 0 & \\
\hline
\end{tabular}




\section{Functional MRI-derived measures and outcomes in vascular lesions}

and Wernicke language centers together, the area of closest language center LAD was not significant. However, the proximity of a vascular lesion to the primary sensorimotor area or Wernicke area was found to be significantly associated with neurological deficits. Nonparametric tests showed that when grouped by Wernicke LAD, there was a trend toward significance, with patients without pretreatment deficits in the $<10-\mathrm{mm}$ and the $10-20 \mathrm{~mm}$ categories more likely to show posttreatment language deficits than those in the $>20-\mathrm{mm}$ category. Additionally, regression analysis showed that in patients with and without pretreatment deficits, there was a trend toward a significant association between distance from vascular lesion to motor activation and posttreatment motor deficits.

These findings are, in part, consistent with previously published studies in patients with brain tumors. For example, Wood et al. ${ }^{20}$ studied patients with primary and metastatic brain tumors and reported a distinctly increasing linear prevalence of motor deficits as LAD decreased. However, they reported that the prevalence of all aphasias decreased dramatically once the LAD of the language area was greater than $1 \mathrm{~cm}$ and leveled off. In contrast to our study, Wood et al. found that the aphasia prevalence trend was only significant for the Broca area LAD and not for the Wernicke area LAD. They hypothesized that proximity to compact eloquent cortical areas was the most important factor, but that white matter involvement could be contributing and confounding the relationship. Language dysfunction through white matter tract damage has been studied in other pathologies but not in AVMs or cavernomas to date. ${ }^{4}$ The extent to which these differences reflect differences in the organization of primary sensorimotor areas compared with language areas is not known. The high variability of anatomical-functional correspondence of language areas has been well reported in the literature in comparison with motor and sensory areas, which have been demonstrated to exhibit greater structural-functional correspondence.

Because a significant relationship between LAD and Broca area aphasia was not observed, it raises questions of language center organization in the setting of complex vascular lesions. For example, Alkadhi et al. ${ }^{1}$ found somatotopic differences in organization of primary motor cortex in close proximity to an AVM; these differences were classified as: 1) functionally displaced independent of direct, structural distortion; 2) activation of motor cortex ipsilateral to the moving limb, in the unaffected hemisphere; or 3) activation in nonprimary motor areas such as the supplemental motor area, and the premotor, cingulate, or parietal regions. Sailor et al. ${ }^{16}$ reported similar findings of shifted supplemental motor area activity contralateral to the unaffected hemisphere in patients with AVMs. For example, a patient with a right frontal AVM that overlapped anatomically with the right supplemental motor area featured left supplemental motor area activity during a left finger-tapping paradigm. In the present study, lesion type affected posttreatment deficits; specifically, patients with cavernomas and not AVMs, who had been deficit free before treatment, showed significant posttreatment language deficits. This may have been due to the wide range of treatments available for patients with AVMs in comparison with patients with cavernomas in our study. It is also possible that the number of patients with AVMs in this study was twice that of patients with cavernomas, and the unequal sample size may have biased the results (Table 7).

This study has several notable limitations. Individualized thresholds were used to counteract the intersubject variability of activation, reduce spurious artifact, and maximize sensitivity and specificity. The same technologist (C.M.) assessed all images. While retrospectively using the individualized threshold maps most closely modeled how fMRI is used in clinical practice today, this nevertheless may have introduced a confounding element of subjectivity in the study. Another limitation is the minimal consideration of white matter tracts, including the arcuate fasciculus or superior longitudinal fasciculus. Lastly, we used all available patient data that satisfied inclusion criteria, and it is possible that because our sample size was not determined by a power analysis, the study could potentially be underpowered and thus insufficiently sensitive to detect some significant differences in outcomes, especially in multivariate analyses. Additionally, in terms of the range of outcomes, we categorized patients in terms of deficits versus no deficits, but did not look at the severity of deficits, which may also have limited our ability to characterize the impact of all of the predictor variables on outcomes.

This study provides valuable information on the prevalence of clinical deficits as a function of LAD to eloquent cortex in patients with AVMs and cavernomas. To some degree, patients can be evaluated before cortical stimulation mapping to provide additional prognostic and management data.

\section{Conclusions}

In the present study we investigated fMRI LAD and its relationship to morbidity in patients with vascular brain lesions (primarily AVMs and cavernomas) preparing to undergo treatment. There was a significant trend between increasing clinical deficits and shorter LAD for sensorimotor and language areas. Additionally, this study also demonstrates the importance of examining deficits by lesion type to obtain a clearer picture of the nature of posttreatment deficits.

\section{Disclosure}

The project described was supported by the University of Wisconsin-Madison, Department of Radiology, Shapiro Program; the University of Wisconsin Institute for Clinical and Translational Research, NIH Pilot Grant No. UL1RR025011, and KL2 Scholar Award; The University of Wisconsin School of Medicine and Public Health Medical Scientist Training Program; AHA Midwest Postdoctoral, Medical Student, and Undergraduate Grants; and a National Institute of Mental Health Challenge Grant (No. RC1MH090912-01).

Author contributions to the study and manuscript preparation include the following. Conception and design: Prabhakaran, Gallagher, Moritz, Meyerand. Acquisition of data: Nair, Young, Holdsworth, Moritz. Analysis and interpretation of data: Prabhakaran, Gallagher, Nair, Regner, Young, Radtke, Pankratz, Holdsworth, Baniulis, Kornder, Voss, Meyerand. Drafting the article: 
Prabhakaran, Gallagher, Nair, Regner, Young, Austin. Critically revising the article: Prabhakaran, Gallagher, Nair, Regner, Young, Austin. Reviewed submitted version of manuscript: Prabhakaran, Nair, Regner. Approved the final version of the manuscript on behalf of all authors: Prabhakaran. Statistical analysis: Gallagher, Nair, Regner, Radtke, Baniulis, Kornder. Study supervision: Prabhakaran.

\section{Acknowledgment}

The authors would like to thank Dr. Hanefi Yildirim for his help during this project, especially in identifying the Spetzler-Martin grade of the AVMs for several of the patients in this study.

\section{References}

1. Alkadhi H, Kollias SS, Crelier GR, Golay X, Hepp-Reymond $\mathrm{MC}$, Valavanis A: Plasticity of the human motor cortex in patients with arteriovenous malformations: a functional MR imaging study. AJNR Am J Neuroradiol 21:1423-1433, 2000

2. Binder JR, Swanson SJ, Hammeke TA, Morris GL, Mueller WM, Fischer M, et al: Determination of language dominance using functional MRI: a comparison with the Wada test. Neurology 46:978-984, 1996

3. Bizzi A, Blasi V, Falini A, Ferroli P, Cadioli M, Danesi U, et al: Presurgical functional MR imaging of language and motor functions: validation with intraoperative electrocortical mapping. Radiology 248:579-589, 2008

4. Breier JI, Hasan KM, Zhang W, Men D, Papanicolaou AC: Language dysfunction after stroke and damage to white matter tracts evaluated using diffusion tensor imaging. AJNR Am J Neuroradiol 29:483-487, 2008

5. Cannestra AF, Pouratian N, Forage J, Bookheimer SY, Martin NA, Toga AW: Functional magnetic resonance imaging and optical imaging for dominant-hemisphere perisylvian arteriovenous malformations. Neurosurgery 55:804-814, 2004

6. Geibprasert S, Pongpech S, Jiarakongmun P, Shroff MM, Armstrong DC, Krings T: Radiologic assessment of brain arteriovenous malformations: what clinicians need to know. Radiographics 30:483-501, 2010

7. Lehéricy S, Biondi A, Sourour N, Vlaicu M, du Montcel ST, Cohen L, et al: Arteriovenous brain malformations: is functional MR imaging reliable for studying language reorganization in patients? Initial observations. Radiology 223:672682,2002

8. Lehéricy S, Duffau H, Cornu P, Capelle L, Pidoux B, Carpentier A, et al: Correspondence between functional magnetic resonance imaging somatotopy and individual brain anatomy of the central region: comparison with intraoperative stimulation in patients with brain tumors. J Neurosurg 92:589-598, 2000

9. Moritz C, Haughton V: Functional MR imaging: paradigms for clinical preoperative mapping. Magn Reson Imaging Clin N Am 11:529-542, 2003

10. Newman GC: Clarification of abc/2 rule for ICH volume. Stroke 38:862, 2007 (Letter)
11. Ogawa S, Lee TM, Kay AR, Tank DW: Brain magnetic resonance imaging with contrast dependent on blood oxygenation. Proc Natl Acad Sci U S A 87:9868-9872, 1990

12. Ogawa S, Lee TM, Nayak AS, Glynn P: Oxygenation-sensitive contrast in magnetic resonance image of rodent brain at high magnetic fields. Magn Reson Med 14:68-78, 1990

13. Pillai JJ: The evolution of clinical functional imaging during the past 2 decades and its current impact on neurosurgical planning. AJNR Am J Neuroradiol 31:219-225, 2010

14. Roessler K, Donat M, Lanzenberger R, Novak K, Geissler A, Gartus A, et al: Evaluation of preoperative high magnetic field motor functional MRI ( 3 Tesla) in glioma patients by navigated electrocortical stimulation and postoperative outcome. J Neurol Neurosurg Psychiatry 76:1152-1157, 2005

15. Sabbah P, Chassoux F, Leveque C, Landre E, Baudoin-Chial S, Devaux B, et al: Functional MR imaging in assessment of language dominance in epileptic patients. Neuroimage 18: 460-467, 2003

16. Sailor J, Meyerand ME, Moritz CH, Fine J, Nelson L, Badie B, et al: Supplementary motor area activation in patients with frontal lobe tumors and arteriovenous malformations. AJNR Am J Neuroradiol 24:1837-1842, 2003

17. Spetzler RF, Martin NA: A proposed grading system for arteriovenous malformations. J Neurosurg 65:476-483, 1986

18. Thickbroom GW, Byrnes ML, Morris IT, Fallon MJ, Knuckey NW, Mastaglia FL: Functional MRI near vascular anomalies: comparison of cavernoma and arteriovenous malformation. J Clin Neurosci 11:845-848, 2004

19. Vikingstad EM, Cao Y, Thomas AJ, Johnson AF, Malik GM, Welch KM: Language hemispheric dominance in patients with congenital lesions of eloquent brain. Neurosurgery 47:562570,2000

20. Wood JM, Kundu B, Utter A, Gallagher TA, Voss J, Nair VA, et al: Impact of brain tumor location on morbidity and mortality: a retrospective functional MR imaging study. AJNR Am J Neuroradiol 32:1420-1425, 2011

21. Yetkin FZ, Mueller WM, Morris GL, McAuliffe TL, Ulmer JL, Cox RW, et al: Functional MR activation correlated with intraoperative cortical mapping. AJNR Am J Neuroradiol 18:1311-1315, 1997

22. Yetkin FZ, Swanson S, Fischer M, Akansel G, Morris G, Mueller W, et al: Functional MR of frontal lobe activation: comparison with Wada language results. AJNR Am J Neuroradiol 19:1095-1098, 1998

Manuscript submitted December 15, 2012.

Accepted February 5, 2013.

Please include this information when citing this paper: DOI: 10.3171/2013.2.FOCUS12417.

Address correspondence to: Vivek Prabhakaran, M.D., Ph.D., University of Wisconsin School of Medicine and Public Health, Department of Radiology, 600 Highland Avenue, Madison, Wisconsin 53792-3252. email: vprabhakaran@uwhealth.org. 